\title{
(6) OPEN ACCESS \\ Efficacy and safety of cannabinoid oromucosal spray for multiple sclerosis spasticity
}

\author{
F Patti, ${ }^{1}$ S Messina, ${ }^{1}$ C Solaro, ${ }^{2}$ M P Amato, ${ }^{3}$ R Bergamaschi, ${ }^{4}$ S Bonavita, ${ }^{5}$ \\ R Bruno Bossio, ${ }^{6}$ V Brescia Morra, ${ }^{7} \mathrm{G} \mathrm{F}$ Costantino, ${ }^{8}$ P Cavalla, ${ }^{9}$ D Centonze, ${ }_{1}^{10,11}$ \\ G Comi, ${ }^{12}$ S Cottone, ${ }^{13}$ M Danni, ${ }^{14}$ A Francia, ${ }^{15}$ A Gajofatto, ${ }^{16} \mathrm{C} \mathrm{Gasperini,}^{17}$ \\ A Ghezzi, ${ }^{18}$ A ludice, ${ }^{19} \mathrm{G} \mathrm{Lus},{ }^{20} \mathrm{G}$ T Maniscalco, ${ }^{21}$ M G Marrosu, ${ }^{22} \mathrm{M}$ Matta, ${ }^{23}$ \\ M Mirabella, ${ }^{24}$ E Montanari, ${ }^{25} \mathrm{C}$ Pozzilli, $_{1}{ }^{26} \mathrm{M}$ Rovaris, ${ }^{27} \mathrm{E} \mathrm{Sessa}_{1}{ }^{28} \mathrm{D}$ Spitaleri, ${ }^{29}$ \\ M Trojano, ${ }^{30} \mathrm{P}$ Valentino, ${ }^{31} \mathrm{M}$ Zappia, ${ }^{1}$ on behalf of the SA.FE. study group
}

For numbered affiliations see end of article.

\section{Correspondence to \\ Dr Francesco Patti, Department "G.F.Ingrassia", Multiple Sclerosis Center, University of Catania, Teaching Hospital Policlinico "G.Rodolico", Via S. Sofia 78, Catania 95123, Italy; patti@unict.it}

FP and SM contributed equally to the manuscript.

Received 28 October 2015 Revised 29 February 2016 Accepted 5 March 2016 Published Online First 9 May 2016

\section{CrossMark}

To cite: Patti F, Messina S, Solaro C, et al. J Neurol Neurosurg Psychiatry 2016;87:944-951.

\section{ABSTRACT}

Background The approval of 9 - $\delta$-tetrahydocannabinol and cannabidiol (THC:CBD) oromucosal spray (Sativex) for the management of treatment-resistant multiple sclerosis (MS) spasticity opened a new opportunity for many patients. The aim of our study was to describe Sativex effectiveness and adverse events profile in a large population of Italian patients with MS in the daily practice setting.

Methods We collected data of all patients starting Sativex between January 2014 and February 2015 from the mandatory Italian medicines agency (AIFA) e-registry. Spasticity assessment by the $0-10$ numerical rating scale (NRS) scale is available at baseline, after 1 month of treatment (trial period), and at 3 and 6 months.

Results A total of 1615 patients were recruited from $30 \mathrm{MS}$ centres across Italy. After one treatment month (trial period), we found $70.5 \%$ of patients reaching a $\geq 20 \%$ improvement (initial response, IR) and $28.2 \%$ who had already reached $a \geq 30 \%$ improvement (clinically relevant response, CRR), with a mean NRS score reduction of $22.6 \%$ (from 7.5 to 5.8 ). After a multivariate analysis, we found an increased probability to reach $I R$ at the first month among patients with primary and secondary progressive MS, $(n=1169$, OR 1.4 $95 \% \mathrm{Cl} 1.04$ to $1.9, p=0.025)$ and among patients with $>8$ NRS score at baseline (OR $1.895 \% \mathrm{Cl} 1.3-2.4$ $\mathrm{p}<0.001)$. During the 6 months observation period, 631 $(39.5 \%)$ patients discontinued treatment. The main reasons for discontinuation were lack of effectiveness $(n=375,26.2 \%)$ and/or adverse events $(n=268,18.7 \%)$. Conclusions Sativex can be a useful and safe option for patients with MS with moderate to severe spasticity resistant to common antispastic drugs.

\section{INTRODUCTION}

Spasticity, defined as abnormally increased muscular tone, is a common symptom in patients with multiple sclerosis (MS) patients. ${ }^{1}$ MS spasticity causes or worsens different MS symptoms, usually having a big impact on patients' quality of life. ${ }^{2}$ The most frequent symptoms associated with spasticity in patients with MS, beyond mobility worsening, are spasms, pain, poor sleep quality (linked to pain and nocturnal spasms) and urinary dysfunction. ${ }^{3}$ Classically recommended medications for generalised spasticity, including baclofen, tizanidine, dantrolene, benzodiazepines and clonazepam, have shown limited clinical benefit in systematic reviews. ${ }^{4} 5$ The approval of 9- $\delta$-tetrahydocannabinol and cannabidiol (THC: CBD) oromucosal spray (Sativex), in a number of European countries, including Italy, provides a new opportunity as an add-on medication for the management of moderate to severe generalised spasticity and related symptoms in patients with MS resistant to common oral antispastic drugs. ${ }^{6} 7$ Sativex is an endocannabinoid system modulator containing $\mathrm{THC}$ and $\mathrm{CBD}$ in a near 1:1 ratio. THC interacts with cannabinoid human receptors that play a key role in the modulation of muscle tone, whereas CBD at higher than natural concentrations may limit the psychoactive effects of THC. ${ }^{8}$ The efficacy of Sativex oromucosal spray as an add-on therapy for symptoms improvement in patients with MS with moderate to severe MS spasticity has been demonstrated in several clinical trials. ${ }^{6-12}$ The largest pivotal phase III, enricheddesign clinical trial included patients with MS with moderate-severe spasticity corresponding to a patient-reported numerical rating scale (NRS) score $\geq 4$.

In this clinical trial, after a first 4 weeks singleblind THC:CBD trial period, 47\% of patients showed an initial improvement in spasticity evaluated by the MS spasticity 0-10 NRS scale (defined as a $\geq 20 \%$ reduction vs baseline). During the second phase, consisting of a double-blinded randomisation of the initial responders to continue either with THC:CBD treatment or placebo, a significantly greater proportion of patients receiving THC:CBD achieved a clinically relevant response (defined as a $\geq 30 \%$ reduction in their baseline NRS score). Almost $47 \%$ of patients reported adverse events (AEs), with mild to moderate dizziness and fatigue being the most common treatment-related adverse event. ${ }^{12}$ Although clinical trials are needed to evaluate the efficacy and safety of a new compound to defend its approval, observational studies are essential to depict the effect of treatment in real-world conditions. ${ }^{13}$ Postapproval surveillance registries have been established to 
evaluate possible short-term and long-term risks associated with Sativex use. ${ }^{14} 15$ The MOVE 2 German observational study $(n>300)$ of THC:CBD oromucosal spray showed results aligned with those from clinical trials, with $42 \%$ of patients being initial responders after 4 weeks. ${ }^{16}$ Another recent prospective observational study carried out in Spain $(n>200)$ provided data about $2 / 3$ of patients still on prescription after 6 months from treatment start. ${ }^{17}$ In these observational studies, patients tended to use lower doses compared with clinical trials (6-7 sprays/day vs $>8$ ) and the incidence of AEs was lower, with no evidence of addiction, abuse, misuse or memory impairment. ${ }^{17}$ Given the growing importance of data derived from real-world observational studies, we decided to collect information about Sativex use in Italy, using the Italian medicine agency (AIFA) prospective e-registry, mandatory for all patients receiving a Sativex prescription in Italy, as a main source document, considering that, after the large sample was collected, much larger than that in the available THC:CBD studies so far, it would bring a comprehensive picture of its effect, minimising biases. For further information, we also used complementarily the involved patients' medical charts retrospective review.

The main aim of our study was to describe Sativex effectiveness and AEs, including possible evidence of abuse or misuse in a large population of Italian patients with MS.

\section{MATERIALS AND METHODS}

\section{Design and setting}

This was a multicentre observational study aimed at collecting prospective reported data to evaluate Sativex effects (effectiveness and tolerability) in a large population of Italian patients with MS with resistant spasticity carried out in a routine outpatient setting. All patients included in the study were treated in accordance with the approved label and expected standards of good clinical practice. Complementary clinical and demographic parameters were acquired retrospectively from the patients' medical records.

The study was approved by the Policlinico-Vittorio Emanuele (Catania, Italy) Ethics Committee (number 37/2015/PO) and by the Ethics Committee of the other participating centres.

Patients were consecutively included in the study at the start of Sativex treatment (baseline) and followed up over 6 months, with data collection at baseline, after 4 weeks (T1), after 12 weeks ( 3 months, T2) and after 24 weeks (6 months, T3) from baseline.

\section{Patient population}

The AIFA registry requests that patients are eligible for starting Sativex treatment when fulfilling the following approved labelrelated inclusion criteria: patients with MS older than 18 years, with moderate to severe spasticity $(0-10$ NRS score $\geq 4)$ and not responding to common and ongoing antispastic drugs (used under their approved label). Exclusion criteria are: severe cardiovascular diseases, history of psychiatric diseases, use of street cannabis and/or other psychoactive drugs and/or MS spasticity NRS score $<4$.

In our study, we collected data from all the patients starting Sativex therapy between 1 January 2014 and end of February 2015 in the participating centres.

\section{Data collection}

We collected data from the AIFA Sativex e-registry website. The MS spasticity evolution was evaluated by the validated $0-10$ NRS patient-rated scale $\left(0=\right.$ no, $10=$ maximal spasticity) ${ }^{18}$ MS physical disability was evaluated using the expanding disability status scale (EDSS). Other parameters such as use of other antispastic drug, previous antispastic drugs and treatment discontinuation were collected.

Furthermore, complementary demographical and clinical history data, tolerability, daily dose (number of puffs per day), clinical response to Sativex, discontinuation reasons and time to discontinuation, and improvement of spasticity-associated symptoms (stiffness, spasms/cramps, clonic movements, sleep disturbances, urinary dysfunctions, pain, depressed mood) were collected from patients' medical charts and using an ad hoc interview. Data were manually entered in an ad hoc created database. The following definitions were used for classifying responses: Sativex effectiveness was evaluated through the initial response (IR) threshold, defined as $\geq 20 \%$ NRS spasticity score improvement versus the baseline value, ${ }^{18}$ and clinically relevant response (CRR) threshold, defined as $\geq 30 \%$ NRS spasticity score improvement versus baseline value. ${ }^{18}$ Tolerability was assessed collecting each $\mathrm{AE}$ and serious adverse event (SAE) occurring during the whole study period, in accordance with the pharmacovigilance regulations.

\section{STATISTICAL ANALYSIS}

Data were analysed using the STATA V.11.0 software packages (StataCorp. 2011. Stata Statistical Software: Release 12. College Station TSL). Data cleaning was performed before the data analysis considering both range and consistence checks.

Quantitative variables were described using means and SDs. The difference between means and the difference between proportions was evaluated by the $t$ test and the $\chi^{2}$ test, respectively. A Shapiro-Wilk test was performed to assess the normal distribution of data. In case of a not normal distribution, appropriate non-parametric tests were performed. Unconditional logistic regression analysis was performed, and for each study variable we calculated ORs, 95\% CIs and $\mathrm{p}$ values (two-tailed $\mathrm{p} \leq 0.05$ significant level). Univariate logistic regressions were fit considering binomial 'IR yes/no' as a dependent variable and age, sex, disease duration, MS type, baseline EDSS and baseline NRS as independent variables. Whenever quantitative variables were dichotomised, the cut-offs were derived using the median. Multivariate analysis was performed to investigate the independent effect of a risk or protective factor after adjustment for one or several other factors or to adjust for confounding variables. Parameters associated with the outcome in the univariate analysis with a threshold of $\mathrm{p}=0.10$ were included in the multivariate model.

\section{RESULTS}

\section{Patient population}

A total of 1615 patients with MS spasticity starting treatment with Sativex were recruited from 30 large Italian MS centres distributed geographically across the nation from about 160 specialised MS all-size centres in the country. Recruited patients started treatment between 1 January 2014 and end of February 2015 (see table 1 for demographic and clinical details). Of the 1615 patients, 18 were excluded from the analyses because baseline NRS was not available. A total of 1432 patients $(89.7 \%$ from baseline) reached the end of the first month trial period $\mathrm{T} 1$ and were eligible for the trial period early response effectiveness analysis. One hundred and sixty-five patients had to be excluded for different reasons (see figure 1). At T2 (3 months), $889(55.6 \%)$ patients and at T3 (6 months), 593 patients $(37.1 \%)$ were included in the analysis (see figure 1$)$. Reasons for not including the remaining patients at T2 and T3 are detailed 


\section{Multiple sclerosis}

Table 1 Clinical and demographic data

\begin{tabular}{llll}
\hline & Total population & RR & SP+PP \\
\hline $\mathrm{N}(\%)$ & 1615 & $315(19.5)$ & $1035+261(80.2)$ \\
Male (\%) & $766(47.4)$ & $147(46.6)$ & $617(47.6)$ \\
Female (\%) & $849(52.6)$ & $168(53.3)$ & $679(52.4)$ \\
Age (years, mean \pm SD) & $51 \pm 9.5$ & $46.1 \pm 8.9$ & $52 \pm 9.5^{*}$ \\
Disease duration (years, mean \pm SD) & $17.5 \pm 8.6$ & $13.4 \pm 7.6$ & $18.4 \pm 8.6^{*}$ \\
Baseline EDSS (median, range) & $6.5(1.5-9.5)$ & $5.0 \pm 1.3$ & $6.7 \pm 0.9^{*}$ \\
NRS score T0, Baseline $(n=1597$ pts, mean $\pm S D)$ & $7.5 \pm 1.4$ & $7.4 \pm 1.5$ & $7.6 \pm 1.4^{* *}$ \\
NRS score T1, month 1 $(n=1432$ pts, mean $\pm S D)$ & $5.9 \pm 1.6$ & $5.5 \pm 1.8$ & $5.9 \pm 1.6$ \\
NRS T2, month 3 $(n=889$ pts, mean $\pm S D)$ & $5.1 \pm 1.6$ & $4.9 \pm 1.8$ & $5.2 \pm 1.5$ \\
NRS T3 month 6 $(n=593$ pts, mean $\pm S D)$ & $4.8 \pm 1.7$ & $4.7 \pm 1.9$ & $4.9 \pm 1.6$ \\
Dose, puffs number T1(mean $\pm S D)$ & $6.8 \pm 2.6$ & $6.2 \pm 2.9$ & $6.8 \pm 2.6$ \\
Dose, puffs number T2 (mean $\pm S D)$ & $6.5 \pm 2.6$ & $6.2 \pm 2.9$ & $6.5 \pm 2.6$ \\
Dose, puffs number T3 (mean $\pm S D)$ & $6.3 \pm 2.8$ & $6.3 \pm 3.1$ & $6.2 \pm 2.7$
\end{tabular}

\section{${ }^{*} \mathrm{p}<0.0001 ;{ }^{* *} \mathrm{p}=0.0251$.}

EDSS, expanding disability status scale; NRS, numerical rating scale; PP, primary progressive; RR, relapsing remitting; SP, secondary progressive.

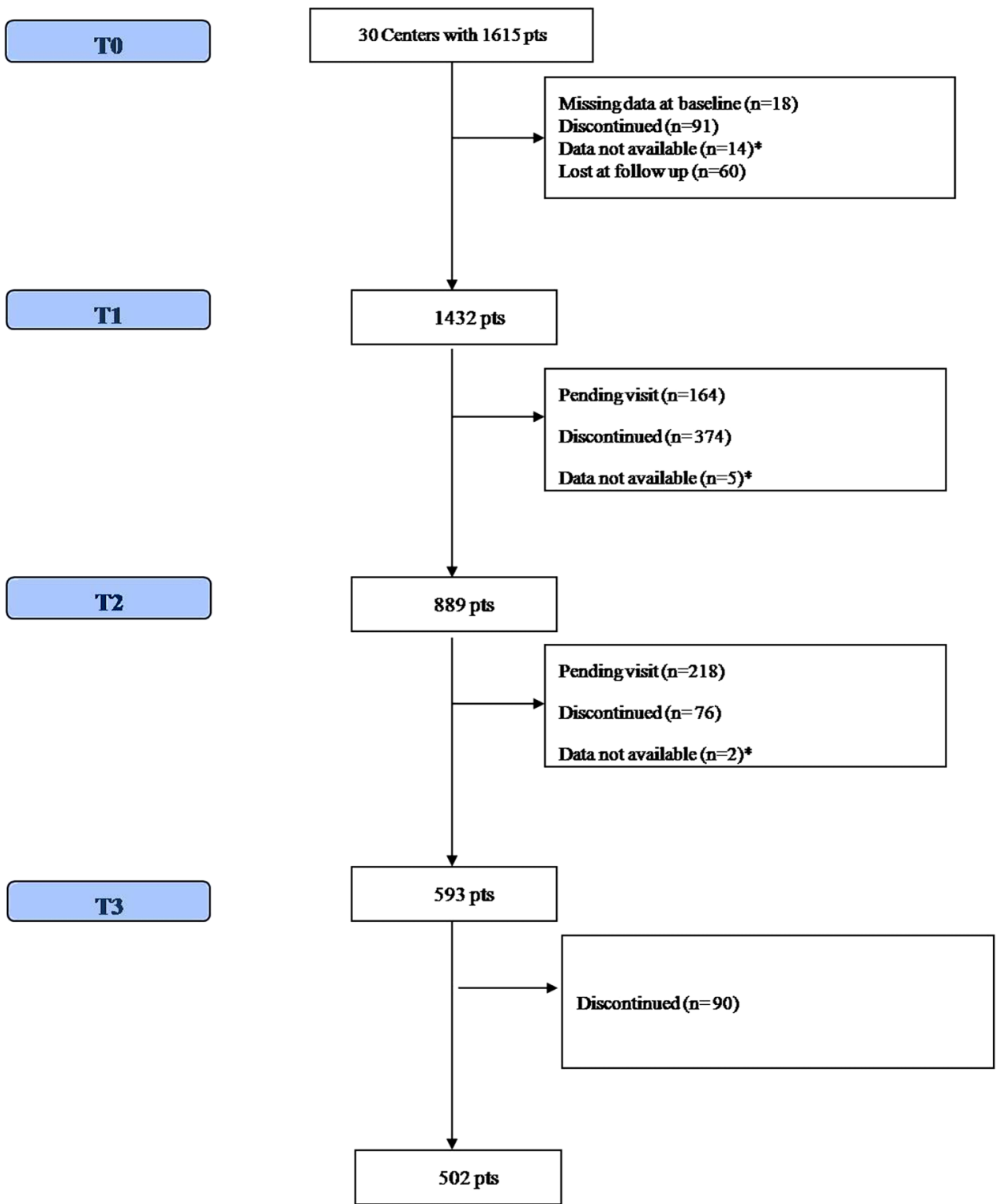

Figure 1 Patient flow chart. *Data not available at the specific time point. 
in figure 1. During the whole observation period, a total of 631 patients $(39.5 \%)$ discontinued therapy.

\section{Effectiveness outcome}

The mean \pm SD 0-10 MS spasticity NRS score value at baseline for the analysable 1597 patients was $7.5 \pm 1.4$.

A statistically significant difference was found comparing the baseline score with the different time points NRS score values of the remaining patients. At T1, the NRS score was $5.9 \pm 1.6$ $(\mathrm{p}<0.0001, \mathrm{n}=1432)$, at T2 5.1 $\pm 1.6(\mathrm{p}<0.0001, \mathrm{n}=889)$ and at T3 $4.8 \pm 1.7(\mathrm{p}<0.0001, \mathrm{n}=593$; see table 1$)$.

Within the 1432 patients who reached T1, end of trial period, 4 weeks after treatment start, a total of 937 patients (65.4\%) were considered clinical responders according to the treating specialist's overall clinical judgement. One hundred and thirty-three patients $(9.3 \%)$ were considered partial responders (suitable to become a responder in longer follow-up), whereas 349 patients $(24.4 \%)$ were deemed to be non-responders. No data were available for 16 patients (1.1\%).

Regarding the NRS scale measurement of the MS spasticity evolution, at T1 1010 patients (70.5\%) reached a $\geq 20 \%$ NRS score reduction compared with baseline (IR threshold), and 405 (28.3\%) had already reached $a \geq 30 \%$ NRS reduction (CRR). We found a $22.6 \%$ reduction of mean NRS score at T1 compared with baseline $(n=1432)$. Within the T1 IR patients subgroup, we found a $30.3 \%$ NRS score mean reduction at T1; and considering T1 CRR patients, we found a $40.8 \%$ NRS score mean reduction at $\mathrm{T} 1$ (figure 2 ).

A total of 889 patients reached the 3 months' visit T2, which is $84.4 \%$ of those who might have reached it on the basis of the treatment start date, $\mathrm{n}=1053$. They showed a mean NRS score of $7.5 \pm 1.5$ at baseline and $5.1 \pm 1.6$ at T2 (32\% mean NRS reduction). A total of 779 patients maintained scores $\geq$ the IR threshold at T2 (88\% of patients reaching this visit and $73.9 \%$ of those who should have reached it), with a mean NRS reduction of $31.6 \%$ at T2 compared with baseline $(7.6 \pm 1.5$ vs 5.2 $\pm 1.4)$. Three-hundred and eleven patients reached the CRR threshold at T2 $35 \%$ of those reaching the visit and $29.5 \%$ of those who should have reached it) with a mean NRS reduction of $41.5 \%$ versus baseline $(7.7 \pm 1.5$ vs $4.5 \pm 1.4)$.

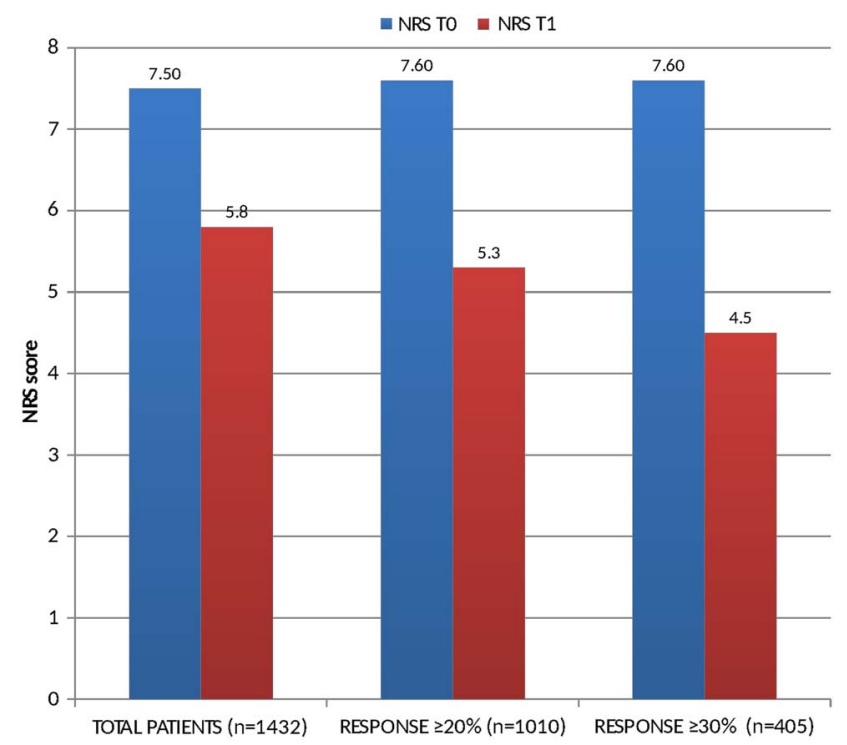

Figure 2 Multiple sclerosis spasticity NRS evolution between T0 and T1. NRS, numerical rating scale.

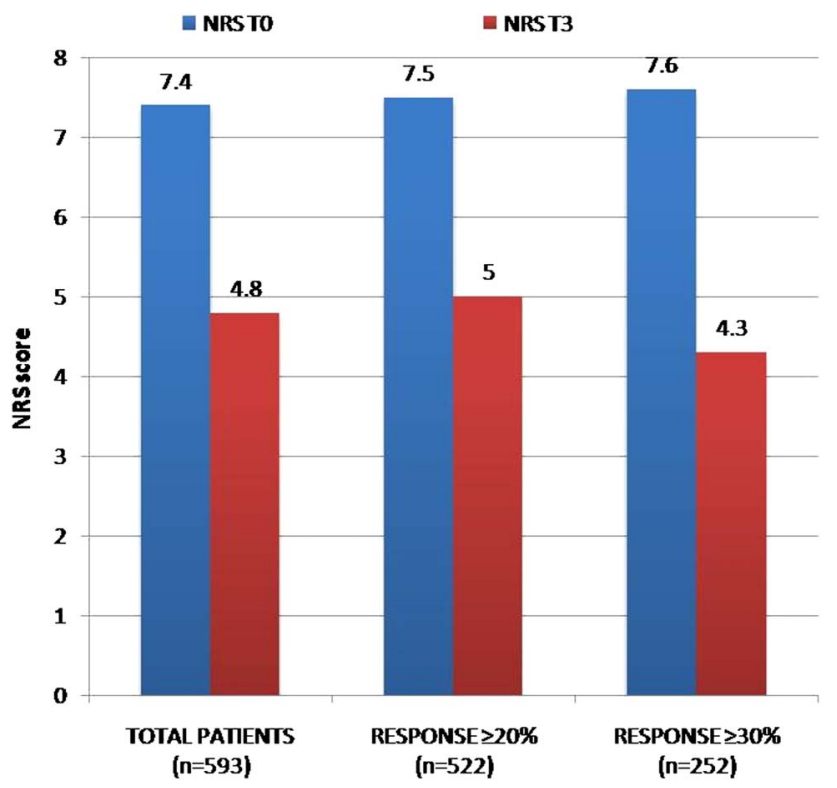

Figure 3 Multiple sclerosis spasticity NRS evolution between T0 and T3. NRS, numerical rating scale.

A total of 593 patients were available for analysis at the T3 sixth months' control visit $73 \%$ of those who might have reached it according to start date, $\mathrm{n}=811$ ). Their mean \pm SD MS spasticity $0-10$ NRS score at T0 was $7.4 \pm 1.5$, and we found a reduction of $35 \%$ at T3 compared with baseline (T3 NRS mean score $4.8 \pm 1.7)$. Five hundred and twenty-two maintained at least a $\geq$ IR threshold score at T3 $(88 \%$ of patients reaching this visit and $64.4 \%$ of those who could have reached it) with a mean NRS reduction of $33.3 \%(7.5 \pm 1.5$ at T0 vs $5 \pm 1.5$ at T3). Considering the CRR population $(n=252,42.5 \%$ of patients reaching this visit and $31 \%$ of those who could have reached it), we found a mean NRS reduction of $43.4 \%(7.6 \pm 1.5$ vs 4.3 \pm 1.5 ; see figure 3 ).

Regarding the medication dose, the mean number of puffs per day was $6.8 \pm 2.6$ at T1 and decreased to $6.5 \pm 2.6$ at T2 $(\mathrm{p}=0.007)$ and $6.3 \pm 2.8$ at T3 $(\mathrm{p}=0.0001$; see table 1$)$.

Univariate analysis logistic regression showed a significant association between baseline NRS score and MS type and the probability to reach IR at T1 (see table 2).

At a multivariate analysis, an age-adjusted and sex-adjusted significant association with MS course and baseline NRS was found. In particular, according to the multivariate analysis, we found an increased probability to reach IR at T1 among patients with secondary progressive primary progressive (SP-PP) (progressive) MS ( $\mathrm{n}=1169$, OR $1.495 \%$ CI 1.05 to $1.86, \mathrm{p}=0.018)$ and among patients with higher spasticity NRS score $(>8)$ at baseline $(\mathrm{n}=358$, OR $1.895 \%$ CI 1.4 to $2.4, \mathrm{p}<0.001$; see table 2$)$.

\section{Reasons for discontinuation}

The number of patients who discontinued therapy during the observation period was 631 (39.5\%). Of them, 396 patients (24.8\% from the overall sample) did not reach the IR threshold. Three hundred and seventy-four patients $(23.4 \%$ of the overall sample) discontinued after the 4-week trial period. Reasons for discontinuation during the whole observation period were (multiple answers possible) lack of effectiveness ( $n=371,23.2 \%$, mostly early), AEs $(n=260,16.3 \%)$, non-adherence $(n=12$, $0.8 \%)$, loss at follow-up $(n=7,0.4 \%)$ patient's choice $(n=5$, $0.3 \%)$ or reasons not available $(n=32,2 \%)$. 
Table 2 Univariate analysis of clinical and demographic predictors of NRS IR at T1 in a population of 1432 patients

\begin{tabular}{|c|c|c|c|c|c|}
\hline \multicolumn{6}{|l|}{ Univariate analysis $\mathrm{T1}$} \\
\hline & $\begin{array}{l}\text { IR+ } \\
\mathrm{N}=1010\end{array}$ & $\begin{array}{l}\mathrm{IR}- \\
\mathrm{N}=422\end{array}$ & OR & $95 \% \mathrm{Cl}$ & p Value \\
\hline \multicolumn{6}{|l|}{ Sex } \\
\hline Women (\%) & $540(53.5)$ & $214(50.7)$ & 1 & NA & NA \\
\hline Men $(\%)$ & $469(46.4)$ & $208(49.3)$ & 0.89 & 0.7 to 1.1 & 0.324 \\
\hline Age (years, mean. Median $=51$ ) & $50.8 \pm 9.6$ & $51.2 \pm 9.6$ & 0.99 & 0.98 to 1.0 & 0.471 \\
\hline $18 / 51$ & $539(53.4)$ & $242(57.3)$ & 1 & NA & NA \\
\hline $51 / 99$ & $471(46.6)$ & $180(42.6)$ & 0.98 & 0.78 to 1.23 & 0.893 \\
\hline Disease duration (years, mean. Median $=17$ ) & $17.8 \pm 8.6$ & $17.4 \pm 8.6$ & 1.0 & 0.99 to 1.01 & 0.416 \\
\hline $0 / 17$ & $518(51.3)$ & $212(50.2)$ & 1 & NA & NA \\
\hline$>17$ & $492(48.7)$ & $210(49.8)$ & 0.93 & 0.74 to 1.17 & 0.552 \\
\hline EDSS (years, mean. Median=6.5) & $6.5 \pm 1.1$ & $6.5 \pm 1.2$ & 1.02 & 0.92 to 1.12 & 0.686 \\
\hline $0-6.5$ & $607(60)$ & $245(58)$ & 1 & NA & NA \\
\hline$>6.5$ & 403 (39.9) & $177(41.9)$ & 0.97 & 0.77 to 1.22 & 0.806 \\
\hline \multicolumn{6}{|l|}{ Multiple sclerosis type } \\
\hline Relapsing remitting & $188(18.6)$ & $74(17.5)$ & 1 & NA & NA \\
\hline Secondary progressive and primary progressive & $822(81.4)$ & $348(82.4)$ & 1.4 & 1.05 to 1.86 & 0.018 \\
\hline NRS (score, mean. Median=8) & $7.6 \pm 1.4$ & $7.3 \pm 1.5$ & 1.2 & 1.1 to 1.3 & $<0.001$ \\
\hline $4 / 8$ & $752(74.5)$ & $322(76.3)$ & 1 & NA & NA \\
\hline$>8$ & $258(25.5)$ & $100(23.7)$ & 1.8 & 1.4 to 2.4 & $<0.001$ \\
\hline
\end{tabular}

EDSS, expanding disability status scale; IR, initial response; NA, not applicable; NRS, numerical rating scale.

Tolerability and safety

AE leading to discontinuation (18.7\% of patients reaching T1, 1432 patients) were basically cognitive/psychiatric disturbances ( $\mathrm{n}=55,3.9 \%$ of patients), in particular, out of the 55 cognitive/ psychiatric AEs, 9 were cognitive ( 3 attention deficit, 3 memory impairment, 3 cognitive worsening) and 46 were psychiatric (32 confusional state, 9 panic attack/anxiety disorder, 3 hallucinations, 1 depressive syndrome, 1 suicidal thoughts). Fatigue $(n=36,2.5 \%)$, drowsiness $(n=32,2.2 \%)$, dizziness $(n=30,2 \%)$, gastrointestinal symptoms $(\mathrm{n}=21,1.4 \%)$, mouth discomfort $(\mathrm{n}=10,0.7 \%)$, allergic reaction $(\mathrm{n}=3,0.2 \%)$ and other neurological symptoms $(n=16,1.1 \%)$ were other reported AEs, all considered drug-related by clinicians. Data were not available for $\mathrm{n}=100(7 \%)$. No abuse, addiction or misuse hints were detected. These AEs were considered drug-related. Of 268 patients, 260 discontinued treatment after the onset of $\mathrm{AE}$ (see table 3). All patients recovered after discontinuation, the

Table 3 AE leading to discontinuation in the population reaching $\mathrm{T} 2$ ( $\mathrm{n}=1432)$

\begin{tabular}{lr}
\hline Adverse events & N (\%) \\
\hline Cognitive disturbances & $9(0.6)$ \\
Psychiatric symptoms & $46(3.2)$ \\
Fatigue & $36(2.5)$ \\
Drowsiness & $32(2.2)$ \\
Dizziness & $30(2.0)$ \\
Gastrointestinal symptoms & $21(1.4)$ \\
Mouth discomfort & $10(0.7)$ \\
Allergic reaction & $3(0.2)$ \\
Other neurological symptoms & $16(1.1)$ \\
Not available & $100(7)$ \\
SAE & $5(0.3)$ \\
\hline AE, adverse event; SAE, serious adverse event. &
\end{tabular}

remaining eight being kept on Sativex treatment. We found five patients $(0.3 \%$ of patients) presenting with a SAE: a hypertensive crisis, one death after acute myocardial infarction, an acute renal failure in a patient with chronic kidney disease, a laryngeal carcinoma case and a breast cancer case. All these SAEs were deemed to be unrelated to the study drug.

\section{DISCUSSION}

In this large multicentre study, we found that $70.5 \%$ of patients reached the IR threshold and that $28.2 \%$ had already reached the CRR threshold at the end of the 4-week trial period. According to the physician's judgement, $65.4 \%$ of patients were considered responders after the first month of treatment, aligning with the proportion of patients reaching IR (70.5\%), whereas $9.3 \%$ were considered partial responders, suitable to become responders in a longer follow-up.

We also found in patients reaching T1 $(\mathrm{n}=1432)$ a $22.6 \%$ mean NRS spasticity score reduction at T1 (first month) and a $35 \%$ mean NRS score reduction at T3 (6 months) compared with baseline, showing a decrease in NRS score from 7.5 at baseline to 5.8 at T1 and 4.8 at T3. Considering the IR population, we found a mean $30.3 \%$ reduction at $\mathrm{T} 1$ and a $33.3 \%$ reduction at T3. Considering the CRR group, we found a $40.8 \%$ reduction at $\mathrm{T} 1$ and $43.4 \%$ reduction at $\mathrm{T} 3$. These data suggest that a meaningful MS spasticity NRS reduction is obtained during the first month and that it is maintained or slightly increased over time (see the T3 6-month follow-up data). This is in line with previous observational studies. ${ }^{16} 1920$ It is noteworthy that the mean number of puffs decreased slightly from 6.8 at T1 to 6.3 at T3, suggesting that effectiveness may be maintained with lower doses compared to clinical trials (mean number of 8.1 puffs/die in clinical trials), ${ }^{12}$ while ruling out the need to increase dose to maintain effect over time (tolerance) and even abuse/misuse trends.

A certainly higher proportion of patients in our study reached the IR $\geq 20 \%$ threshold in the first month (70.4\%) when 
compared with patients from the German MOVE 2 study (41.7\%). However, in the German study, clinicians reported observing clinical relief of resistant spasticity in $74.6 \%$ of patients in the first month of treatment according to their own judgement, in line with the results of our study $(70.4 \%) .{ }^{16}$ In Italy The AIFA e-registry requires an IR threshold attained after the 4- week trial period to continue with the THC:CBD prescription, obtained through an automatic calculation and within a strict time frame. In other countries (Germany included), the NRS improvement is calculated by the clinician without the use of a compulsory web-based registry, thus possibly affecting the perceptions of the initial response. This is confirmed by another recent observational Italian study, showing that a proportion of $71.7 \%$ reached the IR with a mean NRS score reduction of $32 \%$, in line with our results. ${ }^{20}$ The German MOVE 2 study IR patients' subgroup showed a mean reduction of NRS score of $40.2 \%$ at $\mathrm{T} 1$, while in our study a lower mean reduction, $30.3 \%$, was observed. Considering the CRR $\geq 30 \%$ spasticity NRS change versus baseline threshold patients subgroup evolution, a somehow larger mean reduction of $49.9 \%$ was observed at T1 in Germany, ${ }^{16}$ while our results showed a mean reduction of $40.8 \%$ in this subgroup. In a phase 3 randomised placebocontrolled study, participants were also treated with THC:CBD as an add-on therapy in a single-blind manner for 4 weeks. After 4 weeks, $47.5 \%$ of patients reached IR, showing a MS spasticity mean NRS score reduction of $47.6 \%$ compared with baseline NRS, higher than the MOVE 2 observational study score of $41.7 \%$ but still lower than our $70.4 \%$ rate. ${ }^{12}$ Our results showed a higher proportion of IR responders but a smaller mean change in term of NRS between baseline and T1, not fully in accordance with previous studies, although in line with recent findings from an observational Italian study. ${ }^{20}$ This could in part be explained by the AIFA web registry features, not allowing an NRS score $<4$ at baseline and always requiring the IR threshold to be reached at the trial period follow-up visit, thus affecting the recording of the improvement. Indeed, our population seems to be more impaired, in terms of mean EDSS and NRS scores, and has a longer disease duration, when compared with other studies (see table 4). This could in part be explained by multivariate analysis findings showing that reaching the trial period IR threshold could be influenced by a baseline NRS score and disease course (progressive MS). The effectiveness of Sativex on patients with progressive MS spasticity has been investigated by a recent study evaluating clinical and neurophysiological effects of treatment. Although no effect on a neurophysiological measure ( $\mathrm{H}$ reflex) was reported, the study confirmed the clinical benefit (as per the Modified Ashworth scale) in patients with progressive MS, affected by lower limb spasticity. ${ }^{21}$

This finding may in part be related to the higher percentage of SP and PP compared to relapsing remitting in our study population $(80 \% \mathrm{PP}+\mathrm{SP})$, suggesting that patients with progressive MS are more prone to have a spasticity not responding to common anti-spastic drugs, requiring Sativex treatment. Moreover, we found that patients with progressive MS are older, and have a longer disease duration and higher EDSS and NRS at baseline, suggesting that these differences might in part have biased our findings (see table 1). As in our cohort, it was recently reported that severe spasticity is more frequently observed in wheelchair users while patients able to walk $300-500 \mathrm{~m}$ reported a mild to moderate spasticity. ${ }^{7}$ On these grounds, it is conceivable that patients with a higher NRS score and those with progressive MS could be more satisfied with any improvement in their condition, while patients with lower EDSS
Table 4 Sativex in a former observational study and clinical trial versus our study: comparison of key parameters

\begin{tabular}{|c|c|c|c|}
\hline Parameters & $\begin{array}{l}\text { Observational } \\
\text { study }(n=300)^{16}\end{array}$ & $\begin{array}{l}\text { Clinical Trial } \\
(n=572)^{12}\end{array}$ & $\begin{array}{l}\text { Present study } \\
(n=1597)\end{array}$ \\
\hline $\mathrm{N}$ & 300 pts & 572 pts & 1597 \\
\hline Male & 132 & 225 & 766 \\
\hline Female (\%) & $168(60.9)$ & $347(61)$ & $849(52.6 \%)$ \\
\hline Age (years, mean $\pm S D$ ) & $50 \pm 9.4$ & $48.9 \pm 9.63$ & $51 \pm 9.5$ \\
\hline $\begin{array}{l}\text { Disease duration } \\
\text { (years, mean } \pm S D \text { ) }\end{array}$ & $15.4 \pm 9.0$ & $12.4 \pm 7.66$ & $17.5 \pm 8.6$ \\
\hline Baseline EDSS & $6.0(1-9)^{*}$ & $6.0 \pm 1.42 \dagger$ & $6.5(1.5-9.5)^{*}$ \\
\hline NRS T0 in IR (mean $\pm S D)$ & $6.4 \pm 1.8$ & $6.9 \pm 1.2$ & $7.6 \pm 1.4$ \\
\hline NRS T1 in IR (mean \pm SD) & $3.9 \pm 1.5$ & $3.9 \pm 1.5$ & $5.3 \pm 1.3$ \\
\hline $\begin{array}{l}\text { Percentage of reduction } \\
\text { T0-T1 }\end{array}$ & $40.2 \%$ & $43.5 \%$ & $30.3 \%$ \\
\hline IR after 1 month & $42 \%$ & $47 \%$ & $70.5 \%$ \\
\hline CRR after 3 months & $41 \%$ & $36 \%$ & $28.3 \%$ \\
\hline Mean dose (sprays/day) & 6.7 & 8.3 & 6.8 \\
\hline $\begin{array}{l}\text { Adverse events } \\
\text { (1 or more) }\end{array}$ & $15.4 \%$ & $46.9 \%$ & $16.3 \%$ \\
\hline \multicolumn{4}{|c|}{$\begin{array}{l}\text { *Median (min-max). } \\
\text { tMean } \pm \text { SD. } \\
\text { CRR, clinical relevant response ( } 30 \% \text { NRS response); EDSS, expanded disability status } \\
\text { scale; IR, initial response ( } 20 \% \text { NRS response); NRS, numerical rating scale for } \\
\text { multiple sclerosis spasticity. }\end{array}$} \\
\hline
\end{tabular}

scores and thus better walking ability are more demanding in terms of symptoms improvement. Indeed, we should bear in mind that the use of symptomatic treatment is logically mainly based on patients' reported outcomes, rather than on neurological signs examination. However, replication and further analyses are required to better clarify our findings.

About $40 \%$ of our population discontinued treatment, in particular 26.2\% discontinued for lack of effectiveness and 18.7\% for AEs. The most common AEs reported by patients were cognitive/psychiatric effects, fatigue and drowsiness, in line with the tolerability profile reported in other observational studies. ${ }^{14} 1619$

Although this is the largest study reporting Sativex experience in daily clinical practice, our results may be affected by the observational nature of the study. This could in part be mitigated by the use of the AIFA prospective and mandatory eregistry as a source for spasticity NRS information and main patient features. Indeed, in the Italian e-registry, the evaluations are scheduled at 4 weeks from treatment start and then every 3 months, giving us the opportunity to collect data from every centre at the same time points. Another limitation is the lack of spasticity evaluation through the Ashworth healthcare professional impression categorical scale. Although the use of the Ashworth Scale for the assessment of spasticity could be not valid and reliable, results from the German MOVE 2 Study and a recent clinical trial indicated a small but significant improvement. ${ }^{16}$

Finally, the follow-up period duration (up to 6 months) may be insufficient to assess Sativex effectiveness, safety and tolerability long-term effects.

Besides the aforementioned limitations, this study is the largest multicentre observational study so far available evaluating Sativex effectiveness and tolerability profile in clinical practice. Our results confirm Sativex as an effective and safe option for patients with MS with moderate to severe spasticity resistant to common antispastic drugs. Longer and large multicentre follow-up studies are needed to evaluate treatment effectiveness 
and tolerability effect in the population with MS. Further exploitation of the AIFA Sativex e-registry data would be desirable.

\section{Author affiliations}

'Department of Medical, Surgical Science and Advanced Technology "GF Ingrassia", University of Catania, Catania, Italy

${ }^{2}$ Neurology Unit, Department Head and Neck, ASL3 Genova, Genova, Italy

${ }^{3}$ Department NEUROFARBA, University of Florence, Florence, Italy

${ }^{4}$ Department of Neurology, Neurology Institute C Mondino, Pavia, Italy

I Clinic Neurology, II University of Naples, Naples, Italy

${ }^{6}$ Neurology Operating Unit, Multiple Sclerosis Center, Provincial Health Authority of

Cosenza, Cosenza, Italy

${ }^{7}$ Multiple Sclerosis Centre, University Federico II, Naples, Italy

${ }^{8}$ Demyelinating Diseases Centre, Foggia Hospital, Foggia, Italy

${ }^{9}$ Multiple Sclerosis Centre, S. Giovanni Battista, Molinette Hospital, Turin, Italy

${ }^{10}$ Department of Systems Medicine, Multiple Sclerosis Clinical and Research Center,

Tor Vergata University, Rome, Italy

${ }^{11}$ Unit of Neurology and of Neurorehabilitation, IRCCS Neuromed, Pozzilli (IS), Italy

${ }^{12}$ Department of Neurology, San Raffaele Hospital, Milan, Italy

${ }^{13}$ Neuroimmunology Unit, Villa Sofia-Cervello Hospital, Palermo, Italy

${ }^{14}$ Neurology Clinic, Ancona Hospital, Ancona, Italy

${ }^{15}$ Department Neurol Psich, Multiple Sclerosis Center, Sapienza University, Rome, Italy

${ }^{16}$ Multiple Sclerosis Centre, University of Verona, Verona, Italy

${ }^{17}$ Neurology Division, San Camillo Hospital, Rome, Italy

${ }^{18}$ Multiple Sclerosis Centre, Sant'Antonio Abate Hospital, Gallarate, Italy

${ }^{19}$ Multiple Sclerosis Centre, University Hospital Pisa, Pisa, Italy

${ }^{20}$ Multiple Sclerosis Center, Second University of Naples, Naples, Italy

${ }^{21}$ Multiple Sclerosis Centre, Cardarelli Hospital, Naples, Italy

${ }^{22}$ Department of Medical Sciences, University of Cagliari, Cagliari, Italy

${ }^{23}$ Multiple Sclerosis Centre (CRESM), San Luigi Gonzaga Hospital, Orbassano, Italy

${ }^{24}$ Multiple Sclerosis Centre, Cattolica University, Rome, Italy

${ }^{25}$ Multiple Sclerosis Centre, Vaio Hospital, Fidenza, Italy

${ }^{26}$ Multiple Sclerosis Centre, S. Andrea Hospital, Rome, Italy

${ }^{27}$ Multiple Sclerosis Centre, IRCCS Don Gnocchi Foundation, Milan, Italy

${ }^{28}$ Multiple Sclerosis Centre, IRCCS-Bonino Pulejo Centre, Messina, Italy

${ }^{29}$ Multiple Sclerosis Centre, San G. Moscati Hospital, Avellino, Italy

${ }^{30}$ Department of Basic Medical Sciences, Neuroscience and Sense Organs, University

of Bari "Aldo Moro", Bari, Italy

${ }^{31}$ Department of Medical Sciences, Institute of Neurology, University "Magna

Graecia", Catanzaro, Italy

Twitter Follow Ada Francia at @ada.francia@uniroma1.it

Collaborators Benedetti MD, Bertolotto A, Berra E, Bianco A, Buttari F, Cerqua R, Florio C, Fuiani A, Guareschi A, Ippolito D, Nuara A, Palmieri V, Paolicelli D, Petrucci L, Pontecorvo S, Saccà F, Salamone G, Signoriello E, Spinicci G, Russo M, Tavazzi E, Trabucco E, Trotta M, Zaffaroni M.

Contributors FP, SM, CS contributed to the design and drafting for intellectual content of this manuscript. MPA, RB, SB, RBB, VBM, GFC, PC, DC, GC, SC, MD, $A F, A G, C G, A G, A l, G L, G T M, M G M, M M, M M, E M, C P, M R$, ES, DS, MT, PV, MZ and the SA.FE. study group contributed to the drafting for intellectual content of this manuscript.

Competing interests FP has received honoraria for scientific lectures and travel payment from Biogen, Novartis, Teva, Genzyme, Bayer Schering, Merck Serono and Almirall. SM has received honoraria for scientific lectures from Biogen, Almirall, travel payment from Novartis, Biogen Idec, Genzyme, Almirall Bayer Schering, Merck Serono and Teva, and she serves on the scientific advisory board for Biogen. MPA serves on scientific advisory boards for Biogen and Merck Serono and receives speaker honoraria and research support from Biogen Idec, Merck Serono, Novartis, Almirall and Genzyme. AG has received support for travel to scientific meetings from Almirall, Biogen, Genzyme, Merck-Serono and Novartis. RB has received honoraria for scientific lectures and travel payment from Biogen, Novartis, Teva, Genzyme, Bayer Schering, Merck Serono and Almirall. SB received speaker and advisory board honoraria from Biogen, Novartis and Merck-Serono. RBB received a grant for advisory board activities from Almirall. VBM has received honoraria for scientific lectures and travel payment from Biogen, Novartis, Teva, Genzyme, Bayer Schering, Merck Serono and Almirall. DC is an Advisory Board member of Almirall, Bayer Schering, Biogen, Genzyme, GW Pharmaceuticals, Merck-Serono, Novartis and Teva and received honoraria for speaking or consultation fees from Almirall, Bayer Schering, Biogen Idec, Genzyme, GW Pharmaceuticals, Merck Serono, Novartis, Sanofi-Aventis and Teva. He is also an external expert consultant of the European Medicine Agency (EMA), and the principal investigator in clinical trials for Bayer Schering, Biogen Idec, Merck Serono, Mitsubishi, Novartis, Roche, Sanofi-aventis and Teva. His preclinical and clinical research was supported by grants from Bayer,
Biogen, Merck Serono, Novartis e Teva. GC has received honoraria for scientific lectures from Biogen, Novartis, Teva, Genzyme, Bayer Schering, Merck Serono, Almirall. MD has received honoraria for scientific lectures from Novartis, Merck-Serono, Biogen and Teva. AF received a grant for advisory board activities from Almirall. GFC reveived honoraria for speaking activities from Genzyme, Merck-Serono, Biogen and Novartis. CG received honoraria from Biogen, Genzyme, Novartis, Merck Serono, Almirall and Teva. AG received honoraria from Biogen, Genzyme, Novartis, Merck Serono, Almirall and Teva. GL received honoraria from Biogen, Genzyme, Novartis, Merck Serono, Almirall and Teva. GTM received speaking and advisory honoraria from Biogen, Novartis and Teva. MGM has received speaker honoraria and honoraria for serving on advisory board activities from Bayer Schering, Biogen Idec, Merck Serono, Novartis, Sanofi Aventis and Teva, and research grants from Merck Serono and Novartis. MM received honoraria for serving on the scientific advisory boards of Almirall, Novartis, Biogen and Genzyme. MM received honoraria from Biogen, Genzyme, Novartis, Merck Serono, Almirall and Teva. EM received honoraria from Biogen, Genzyme, Novartis, Merck Serono, Almirall and Teva. CP received honoraria for scientific lectures from Biogen, Novartis, Teva, Genzyme, Bayer Schering, Merck Seronoand Almirall. MR received honoraria from Biogen, Genzyme, Novartis, Merck Serono, Almirall and Teva. ES received honoraria from Biogen, Genzyme, Novartis, Merck Serono, Almirall and Teva. MT received honoraria for scientific lectures from Biogen Idec, Novartis, Teva, Genzyme, Bayer Schering, Merck Serono and Almirall. MZ has received compensation for consulting services from Boehringer-Ingelheim, Lundbeck and Union ChimiqueBelge, and scientific grants from AIFA- Agenzia Italiana del Farmaco, Novartis and Lundbeck CS received honoraria from Biogen, Genzyme, Novartis, Merck Serono, Almirall and Teva.

Ethics approval The study was approved by the Policlinico-Vittorio Emanuele (Catania, Italy) Ethics Committee ( $n^{\circ}$ 37/2015/PO) and by the Ethics Committee of the other participating centres.

Provenance and peer review Not commissioned; externally peer reviewed.

Open Access This is an Open Access article distributed in accordance with the Creative Commons Attribution Non Commercial (CC BY-NC 4.0) license, which permits others to distribute, remix, adapt, build upon this work non-commercially, and license their derivative works on different terms, provided the original work is properly cited and the use is non-commercial. See: http://creativecommons.org/ licenses/by-nc/4.0/

\section{REFERENCES}

1 Arroyo R, Vila C, Clissold S. Retrospective observational study of the management of multiple sclerosis patients with resistant spasticity in Spain: the ' $5 \mathrm{E}^{\prime}$ study. Expert Rev Pharmacoecon Outcomes Res 2011;11:205-13.

2 Rizzo MA, Hadjimichael OC, Preiningerova J, et al. Prevalence and treatment of spasticity reported by multiple sclerosis patients. Mult Scler 2004;10:589-95.

3 Pozzilli C. Overview of MS spasticity. Eur Neurol 2014;71(Suppl 1):1-3.

4 Beard S, Hunn A, Wight J. Treatments for spasticity and pain in multiple sclerosis: a systematic review. Health Technol Assess 2003;7:iii, ix-x, 1-111.

5 Shakespeare DT, Boggild M, Young C. Anti-spasticity agents for multiple sclerosis. Cochrane Database Syst Rev 2003;(4):CD001332.

6 Collin C, Davies P, Mutiboko IK, et al, Sativex spasticity in MSSG. Randomized controlled trial of cannabis-based medicine in spasticity caused by multiple sclerosis. Eur J Neurol 2007;14:290-6.

7 Meuth SG, Vila C, Dechant KL. Effect of Sativex on spasticity-associated symptoms in patients with multiple sclerosis. Expert Rev Neurother 2015;15:909-18.

8 Perez J. Combined cannabinoid therapy via an oromucosal spray. Drugs Today (Barc) 2006:42:495-503.

9 Wade DT, Makela P, Robson P, et al. Do cannabis-based medicinal extracts have general or specific effects on symptoms in multiple sclerosis? A double-blind, randomized, placebo-controlled study on 160 patients. Mult Scler 2004;10:434-41.

10 Wade DT, Makela PM, House $\mathrm{H}$, et al. Long-term use of a cannabis-based medicine in the treatment of spasticity and other symptoms in multiple sclerosis. Mult Scler 2006:12:639-45

11 Collin C, Ehler E, Waberzinek G, et al. A double-blind, randomized, placebo-controlled, parallel-group study of Sativex, in subjects with symptoms of spasticity due to multiple sclerosis. Neurol Res 2010;32:451-9.

12 Novotna A, Mares J, Ratcliffe $S$, et al. A randomized, double-blind, placebo-controlled, parallel-group, enriched-design study of nabiximols* (Sativex®), as add-on therapy, in subjects with refractory spasticity caused by multiple sclerosis. Eur J Neurol 2011;18:1122-31.

13 Fernández 0 . Advances in the management of MS spasticity: recent observational studies. Eur Neurol 2014;72(Suppl 1):12-14.

14 Eltayb A, Etges T, Wright S. An observational post-approval registry study of patients prescribed Sativex $®$. Results from clinical practice. [Poster 1041]. Mult Scler 2013;19(Suppl 1):74

15 Koehler J, Feneberg W, Meier M, et al. Clinical experience with THC:CBD oromucosal spray in patients with multiple sclerosis-related spasticity. Int I Neurosci 2014;124:652-6 
16 Flachenecker P, Henze T, Zettl UK. Nabiximols (THC/CBD oromucosal spray, Sativex() in clinical practice-results of a multicenter, non-interventional study (MOVE 2) in patients with multiple sclerosis spasticity. Eur Neurol 2014;71:271-9.

17 Celia Oreja-Guevara BC, Manuel Ordás C, Vila C, et al. Observational safety study of THC:CBD oromucosal spray (Sativex) in multiple sclerosis patients with spasticity. Clin Exp Pharmacol 2015;5:184.

18 Farrar JT, Troxel AB, Stott C, et al. Validity, reliability, and clinical importance of change in a 0-10 numeric rating scale measure of spasticity: a post hoc analysis of a randomized, double-blind, placebo-controlled trial. Clin Ther 2008;30:974-85.
19 Flachenecker $\mathrm{P}$, Henze T, Zettl UK. Long-term effectiveness and safety of nabiximols (tetrahydrocannabinol/cannabidiol oromucosal spray) in clinical practice. Eur Neurol 2014;72:95-102.

20 Ferrè L, Nuara A, Pavan G, et al. Efficacy and safety of nabiximols (Sativex) on multiple sclerosis spasticity in a real-life Italian monocentric study. Neurol Sci 2016;37:235-42.

21 Leocani L, Nuara A, Houdayer E, et al. Sativex and clinical-neurophysiological measures of spasticity in progressive multiple sclerosis. J Neurol 2015;262: 2520-7. 